\title{
Optimization of Asymmetric Acceleration Waveform for Haptic Device Driven by Two-Degree-of-Freedom Oscillatory Actuator
}

\author{
Masayuki Kato ${ }^{* a)}$ Student Member, Junya Nitta* Associate \\ Katsuhiro Hirata* Senior Member
}

(Manuscript received May 27, 2015, revised Aug. 28, 2015)

\begin{abstract}
Mobile haptic devices are being studied extensively because they have great potential for use as information transfer devices. Amemiya et al. proposed a method for haptic feedback with a slider-crank mechanism that uses an asymmetrical drive. We have recently proposed a haptic device that uses a small two degree-of-freedom (2-DOF) oscillatory actuator. Using this actuator, we were able to reproduce the same asymmetric acceleration as the one achieved by Amemiya. However this waveform is dependent on the mechanical structure of the slider-crank mechanism and thus it is not necessary to use it for our actuator. In this paper, first, the relationship of the properties of the asymmetric acceleration to haptic perception is investigated by conducting experiments on volunteers. Next, by applying a genetic algorithm to the optimization method, an optimized asymmetric acceleration waveform is created. The effectiveness of the method is demonstrated by the power consumption of our actuator and the ratio of correct answers given by the volunteers.
\end{abstract}

Keywords: asymmetric acceleration, finite-element methods, haptic devices, genetic algorithm, two degree of freedom (2-DOF) oscillatory actuator

\section{Introduction}

Linear oscillatory actuators (LOAs) have been used in a wide range of applications because they have a lot of advantages: high efficiency, simple structure, easy control, and so forth $^{(1)-(4)}$. Small-sized LOAs have a great potential to be used as handheld haptic devices in the near future, e.g. entertainment application or walking navigation for visually impaired person.

Haptic devices that employ vibration ${ }^{(5)(6)}$ have been studied. Amemiya et al. proposed a method for haptic feedback with a slider-crank mechanism that uses asymmetrical drive $^{(7)(8)}$. Haptic devices employing asymmetric acceleration can create the illusion of a pulling sensation without needing a grounded structure because human perception is nonlinear. However, these devices are not suitable for mobile applications because of their size. Furthermore it can only produce a one-DOF force, and also generates unnecessary torque due to the rotation of the connecting rod.

We have proposed a 2-DOF oscillatory actuator ${ }^{(9)(10)}$ and succeeded in creating an acceleration waveform that is similar to the one generated by the slider-crank mechanism. However, it was then realized that the slider-crank waveform is unique and specific to the mechanical structure of the slidercrank mechanism. Therefore it is actually not necessary to use it for our 2-DOF actuator, which can synthesize a wide range of acceleration waveforms. In a previous paper, we

a) Correspondence to: Masayuki Kato. E-mail: masayuki.kato@ ams.eng.osaka-u.ac.jp

* Department of Adaptive Machine Systems, Graduate School of Engineering, Osaka University

2-1, Yamadaoka, Suita, Osaka 565-0871, Japan proposed a new acceleration waveform that consumed less power $^{(11)}$.

However, the asymmetric acceleration waveform shown in Ref. (11) is not necessarily the best waveform since it has not been optimized. In this paper, first, how the properties of the asymmetric acceleration relate to haptic perception is investigated by conducting experiments on volunteers. Then, by applying a genetic algorithm to the optimization method, an optimized asymmetric acceleration waveform is created. Finally, the effectiveness of the optimized acceleration waveform is verified through 3-D finite element analysis and also experiments on volunteers.

\section{Basic Structure and Operating Principle}

The basic structure of the 2-DOF oscillatory actuator in this study is shown in Fig. 1. This actuator mainly consists of a stator, mover and four springs. The external dimensions of the mover are $30 \mathrm{~mm} \times 30 \mathrm{~mm} \times 8 \mathrm{~mm}(\mathrm{~L} \times \mathrm{W} \times \mathrm{H})$

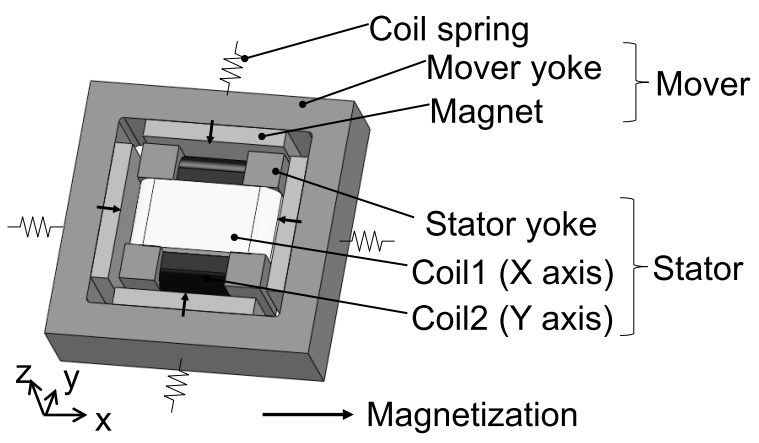

Fig. 1. Basic structure of 2-DOF oscillatory actuator 


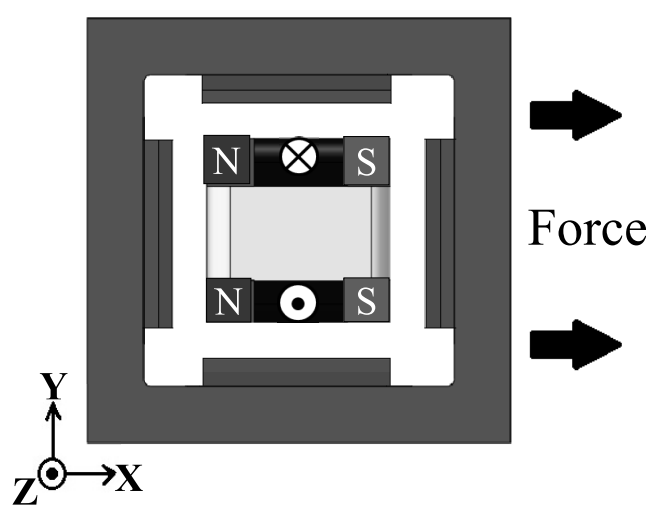

Fig. 2. Operating Principle

respectively. The air gap is 1 millimeter in all dimensions when the mover is at the center position. The mover is assumed to move with a range of \pm 0.5 millimeters in both the $x$-axis and $y$-axis directions. The total mass is $118 \mathrm{~g}$.

The operating principle is shown in Fig. 2. When Coil 1 is excited, a magnetic pole is induced and the mover moves in the $x$-axis direction due to magnetic attraction and repulsion forces. A force in the opposite direction is generated when the direction of the current is reversed. Exciting Coil 2 moves the mover in the $y$-axis.

\section{Analysis Method}

3.1 Magnetic Field Analysis Using the magnetic vector potential $\boldsymbol{A}$ and the current flowing through the coils $I$, the equations of the magnetic field and electric current are coupled and are expressed as follows:

$$
\begin{aligned}
& \operatorname{rot}(v \operatorname{rot} \boldsymbol{A})=\boldsymbol{J}_{0}+v_{0} \operatorname{rot} \boldsymbol{M} \\
& E=V_{0}-R I-\frac{d \phi}{d t} \ldots \ldots \ldots \\
& \boldsymbol{J}_{0}=\frac{n_{c}}{S_{c}} \boldsymbol{I n}_{s} \ldots \ldots \ldots \ldots
\end{aligned}
$$

where $v$ is the reluctivity, $\boldsymbol{J}_{0}$ is the excitation current density, $\boldsymbol{v}_{0}$ is the reluctivity of vacuum, $\boldsymbol{M}$ is the magnetization of the permanent magnet, $V_{0}$ is the applied voltage, $R$ is the resistance, $\phi$ is the interlinkage magnetic flux of the excited coil, $\boldsymbol{n}_{s}$ is the unit normal vector of the cross section of the coil, and $n_{\mathrm{c}}$ and $S_{\mathrm{c}}$ are the number of turns and the cross sectional area of the coil, respectively.

3.2 Coupled Analysis with Motion Equation motion equation of the mover is expressed as follows:

$$
\begin{aligned}
& M_{x} \frac{d^{2} x}{d t^{2}}+D_{x} \frac{d x}{d t}+k_{x} x=F_{x} \\
& M_{y} \frac{d^{2} y}{d t^{2}}+D_{y} \frac{d y}{d t}+k_{y} y=F_{y} .
\end{aligned}
$$

Where $M_{x}$ and $M_{y}$ are the masses of the mover, which also include the respective masses of the support structures. $x$ and $y$ are the displacements of the mover in the $\mathrm{x}$-axis and $\mathrm{y}$-axis. $D_{x}$ and $D_{y}$ are the viscous damping coefficients, $F_{x}$ and $F_{y}$ are the electromagnetic forces acting on the mover, and $k_{x}$ and $k_{y}$ are the spring constants in the $x$-axis and $y$-axis, respectively.

The thrust of the mover is calculated by the Maxwell stress tensor method, and is substituted into (4) and (5). The position of the mover is calculated at each time step. The applied

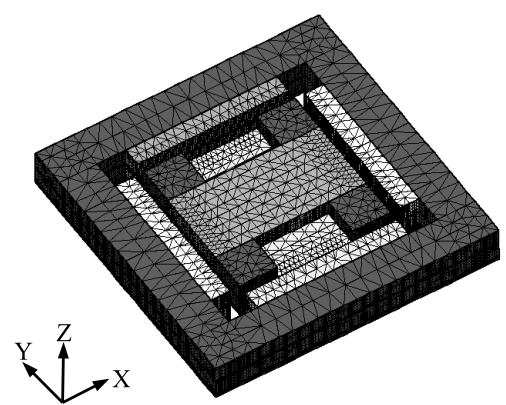

Fig. 3. FEM model for analysis ( $1 / 2$ region)

Table 1. Analysis condition

\begin{tabular}{c|c|c}
\hline Magnets & Magnetization of magnets $[\mathrm{T}]$ & 1.42 \\
\hline \multirow{4}{*}{ Coils } & Coil 1 (X-axis) Resistance $[\Omega]$ & 5.02 \\
\cline { 2 - 3 } & Coil 2 (Y-axis) Resistance $[\Omega]$ & 5.40 \\
\cline { 2 - 3 } & Number of turns [turn] & 193 \\
\hline \multirow{3}{*}{$\begin{array}{l}\text { X-axis } \\
\text { Y-axis }\end{array}$} & Mass of mover [g] & 64.5 \\
\cline { 2 - 3 } & Mass of stator [g] & 52.0 \\
\cline { 2 - 3 } & Spring constant $[\mathrm{N} / \mathrm{m}]$ & 74.9 \\
\hline
\end{tabular}

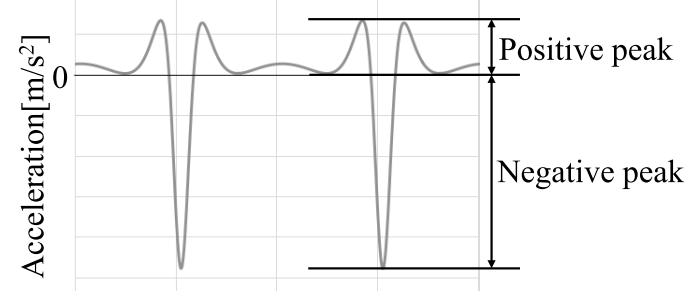

Time[s]

Fig. 4. Properties of asymmetric acceleration waveform

Table 2. Subject experiment condition

\begin{tabular}{c|c}
\hline Number of tests & 25 times each hand \\
\hline Correct answer feedback & None \\
\hline Number of output direction & 4 \\
\hline Operating time $(\mathrm{sec})$ & $2 \sim 3$ \\
\hline Interval time $(\mathrm{sec})$ & 1 \\
\hline
\end{tabular}

voltage for asymmetric acceleration drive is calculated using MATLAB/Simulink ${ }^{(10)}$.

3.3 Analysis Model and Condition Figure 3 shows the FEM model without the air region. The analyzed region is $1 / 2$ of the whole region because of the symmetry. The analysis conditions during dynamic analysis are shown in Table 1. The spring constant was configured so that the resonance frequency is larger than the operating frequency.

\section{Required Acceleration Characteristics}

In this section, the effects of the properties of the asymmetric acceleration on haptic perception were determined through experiments on volunteers. Two effects on haptic perception were studied using the acceleration waveform shown in Fig. 4.

4.1 Subject Experiment Method Table 2 shows the conditions of the experiments on volunteers. Ten normal healthy adults (nine men and one woman) aged between 22 


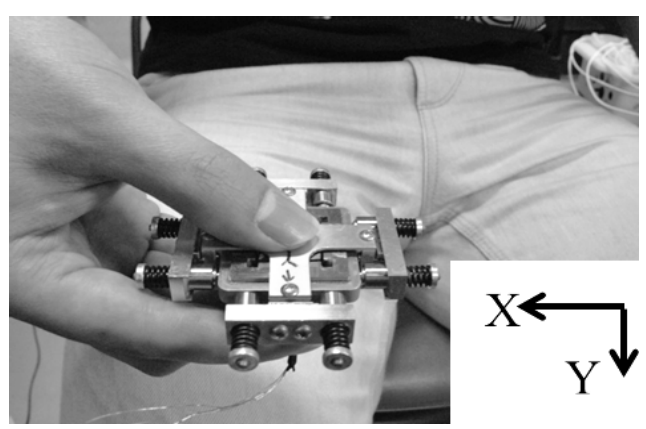

Fig. 5. Subject holding our 2-DOF oscillatory actuator

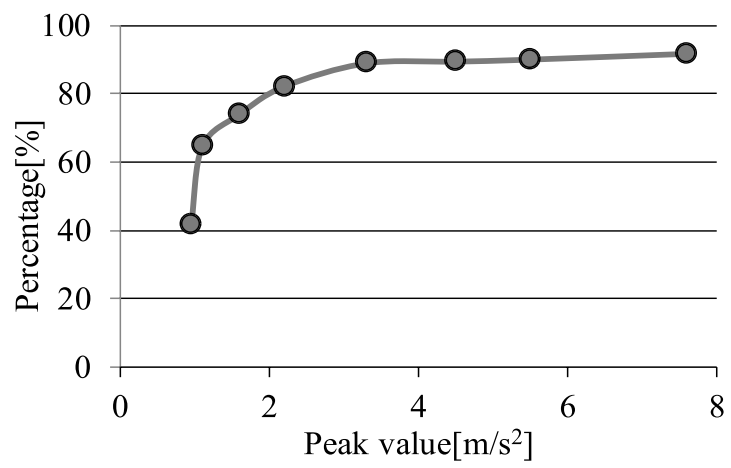

Fig. 6. Relationship between the negative peak and the percentage of correct answers

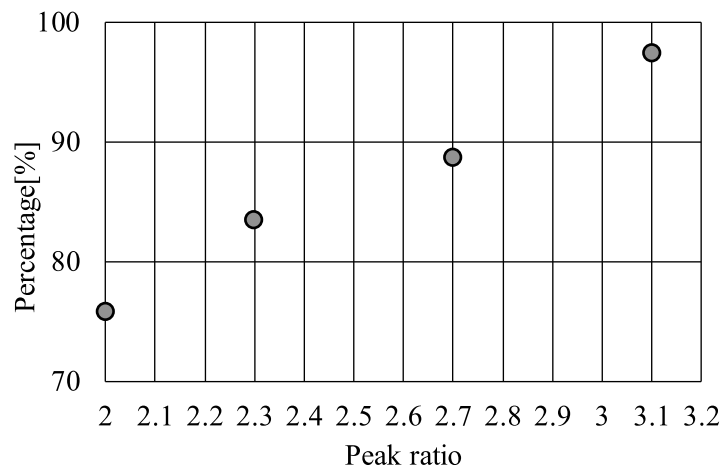

Fig. 7. Relationship between the ratio of the positive to the negative peaks and the percentage of correct answers

and 28 years participated in this experiment. All of them reported that they were right-handed. They were blindfolded and their ears were covered with headphones, and they were told to hold the device as shown in Fig. 5. In this state, the volunteers can receive only tactile information. Next, our 2DOF actuator was driven in four directions, left, right, front and back, selected at random. After each turn, the volunteers had to indicate the direction that they felt the actuator move.

4.2 Results and Discussion Figure 6 shows the relationship between the size of the negative acceleration peak and the percentage of correct answers, with the ratio of the positive to the negative peaks being constant. As the peak value increases, the percentage of correct answers also increases. The percentage of correct answers seems to saturate when the percentage of correct answers are about $4.5 \mathrm{~m} / \mathrm{s}^{2}$. Figure 7 shows the relationship between the ratio of the positive to the negative peaks and the percentage of correct answers. The percentage of correct answers increased linearly with the ratio. From these results, we determined that the ra- tio of the negative to the positive peaks needs to be as large as possible and the negative acceleration peak should be more than $4.5 \mathrm{~m} / \mathrm{s}^{2}$ to achieve a high percentage of correct answers.

\section{Acceleration Waveform Optimization}

5.1 Optimization Method In this section, the method to achieve the required acceleration characteristics described in the previous section is explained. The problem that needs to be solved is a multiple objective optimization problem because the peak ratio has to be maximized but at the same time the power consumption has to be minimized. The objective function is therefore given as follows:

$$
E(R, P)=\frac{R}{R_{t}}+\frac{P_{t}}{P}
$$

where $E$ is the evaluation value of the objective function, $R$ is the ratio of the positive to the negative acceleration peaks, $R_{\mathrm{t}}$ is the target ratio, and $P$ is the power consumption, $P_{\mathrm{t}}$ is the target power consumption. Power consumption is calculated as follows:

$$
P=\frac{1}{T} \int R I^{2} d t
$$

where $T$ is the period of the oscillation. The negative acceleration peak is set to be more than $4.5 \mathrm{~m} / \mathrm{s}^{2}$ and the amplitude of the mover is set to be less than $0.5 \mathrm{~mm}$. In this paper, the objective function is maximized. The target acceleration used in this optimization is given as follows:

$$
a(t)=\sum_{k=1}^{N}\left(A_{k} \cos (2 \pi f t)\right)
$$

where $a(\mathrm{t})$ is the acceleration, $f$ is the frequency of the oscillation, $t$ is time, $N$ is the upper bound of the harmonic order, and $A_{\mathrm{k}}$ is the weight coefficient of each harmonic order that is used as an optimization parameter. In this study, the value of $A_{\mathrm{k}}$ is optimized through a genetic algorithm (GA). The flowchart for the acceleration waveform optimization using GA is shown in Fig. 8 and optimization condition is shown in Table 3.

5.2 Optimization Result and Discussion Figure 9 shows the change in the evaluation value of the objective function when acceleration optimization using GA was performed. Although the evaluation value seems to fluctuate wildly up to the 30th generation, it successfully increased and reaches its termination at about the 230th generation. Figure 10 shows the optimized values of $A_{k}$.

Next, FEM analysis using the optimized waveform was conducted. First, Fig. 11 shows the calculated results of the steady-state mover acceleration, displacement and voltage when the $x$-axis was operated at a frequency of $10 \mathrm{~Hz}$ under the acceleration waveform shown in Ref. (9). From Fig. 11, an asymmetric acceleration can be observed. The negative acceleration peaks are $7.9 \mathrm{~m} / \mathrm{s}^{2}$ and the ratio of the positive to the negative acceleration peaks are 2.47. Power consumption was 3.2 W. Next, Fig. 12 shows the calculated results of the steady-state mover acceleration, displacement, and voltage when the $x$-axis was operated at a frequency of $10 \mathrm{~Hz}$ under the optimized acceleration waveform. From Fig. 12, an asymmetric acceleration can also be observed. The negative 


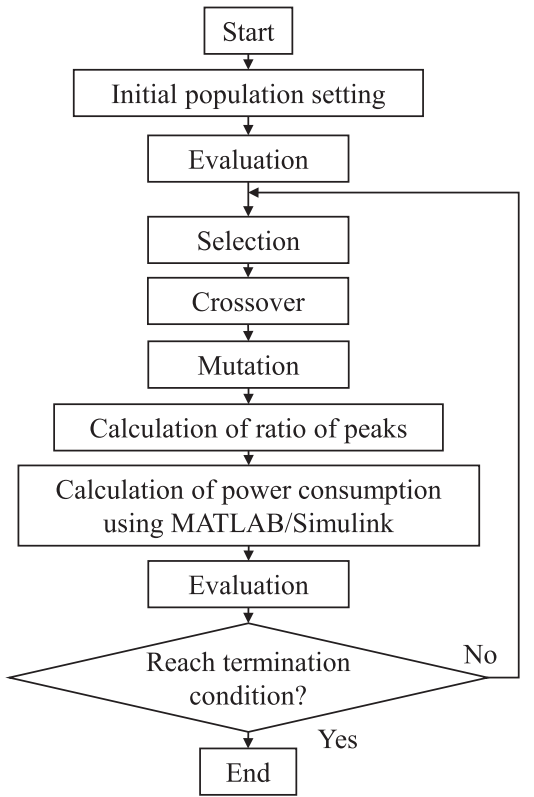

Fig. 8. Flowchart for acceleration waveform optimization

Table 3. Optimization condition

\begin{tabular}{c|c}
\hline $\mathrm{N}$ & 15 \\
\hline Number of population & 60 \\
\hline Crossover method & Uniform crossover \\
\hline Crossover probability & 0.5 \\
\hline Mutation probability & 0.06 \\
\hline Number of maximum generation & 500 \\
\hline
\end{tabular}

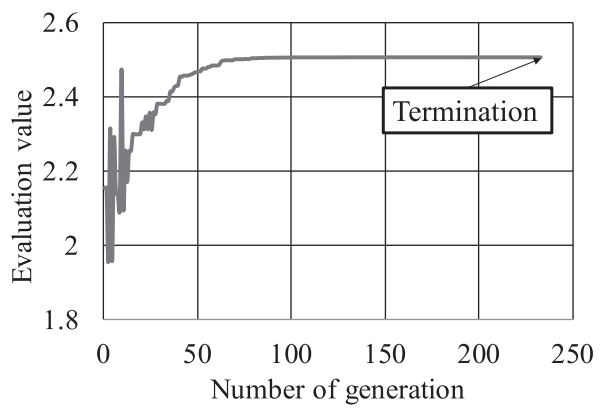

Fig. 9. Change in evaluation value

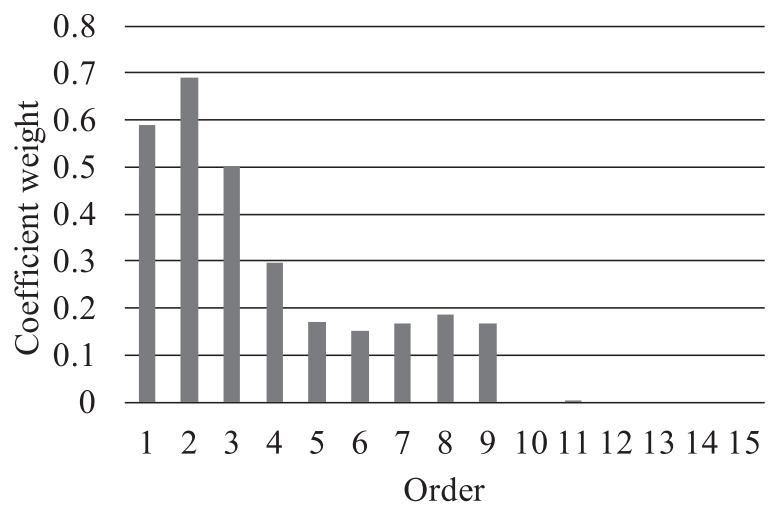

Fig. 10. Optimized weight coefficient

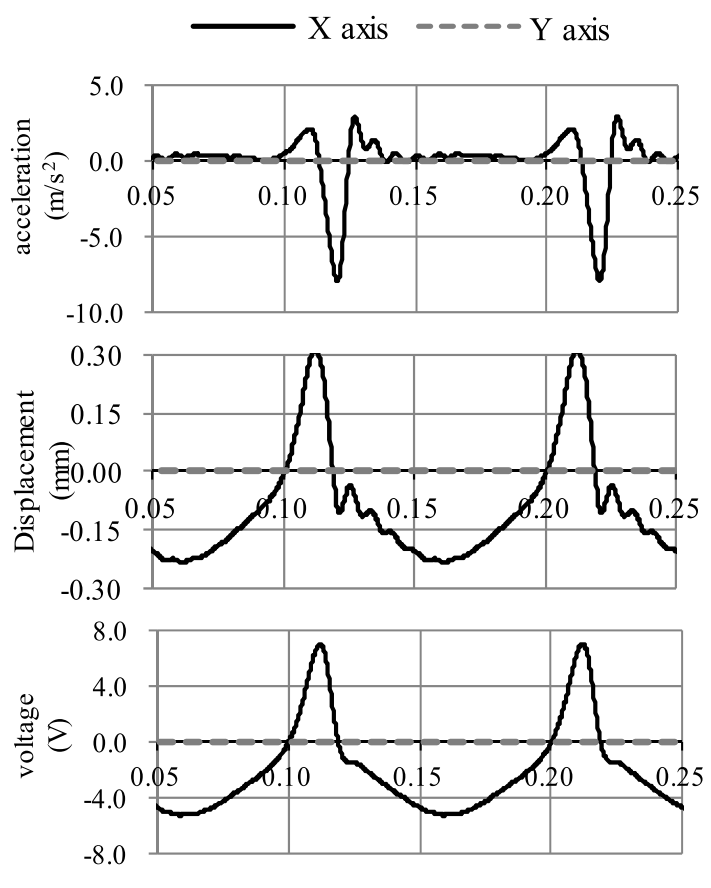

time(s)

Fig. 11. Dynamic analysis results before optimization

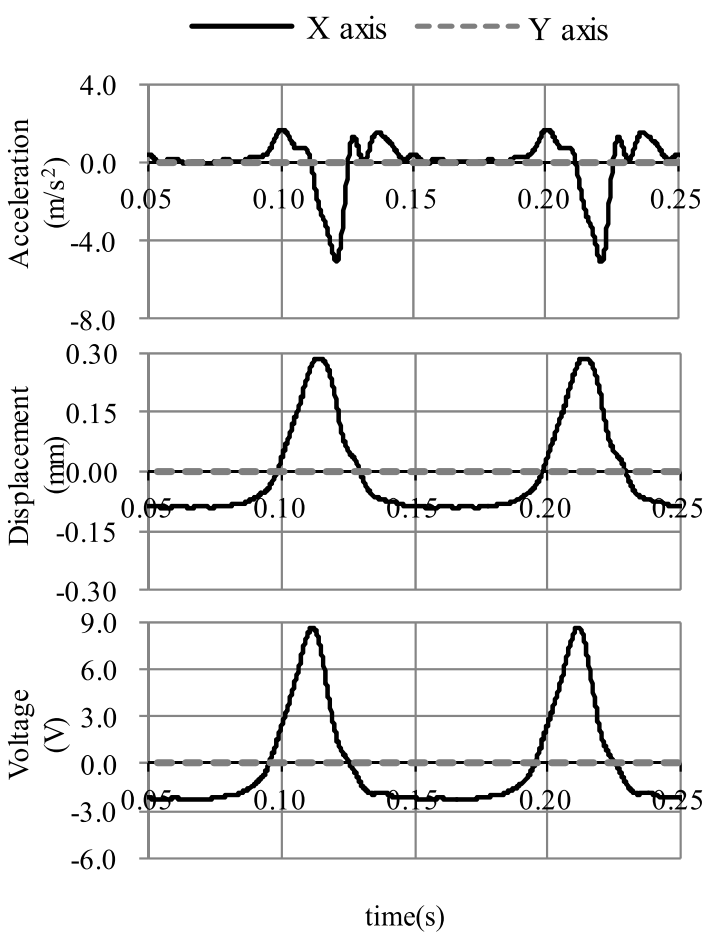

Fig. 12. Dynamic analysis results after optimization

acceleration peaks are $5.1 \mathrm{~m} / \mathrm{s}^{2}$ and the ratio of the positive to the negative acceleration peaks are 2.97, which is larger than that shown in Fig. 11. Power consumption was $1.68 \mathrm{~W}$, which is lower than that shown in Fig. 11 by $47 \%$. This proves the effectiveness of the acceleration optimization method and the optimized acceleration waveform.

5.3 Experimental Verification Next, measurements using our 2-DOF oscillatory actuator were conducted. Figure 13 shows the experimental setup. Actual acceleration 


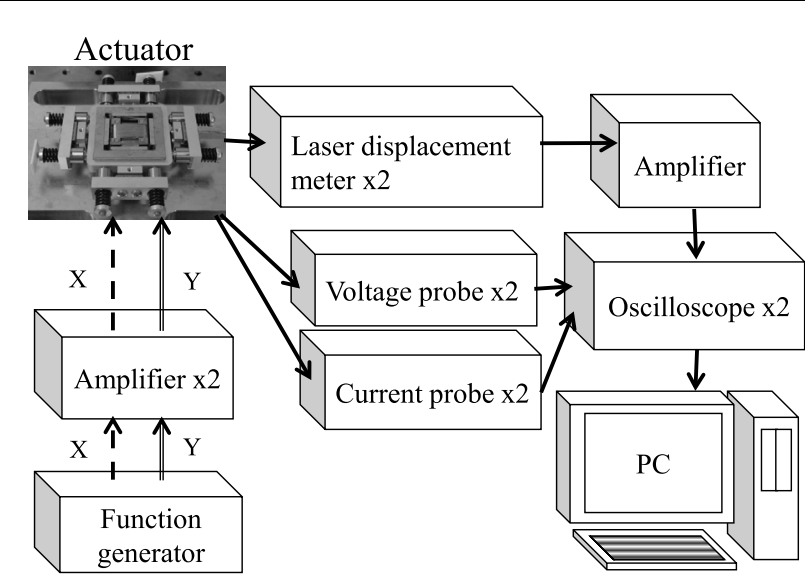

Fig. 13. Experimental setup
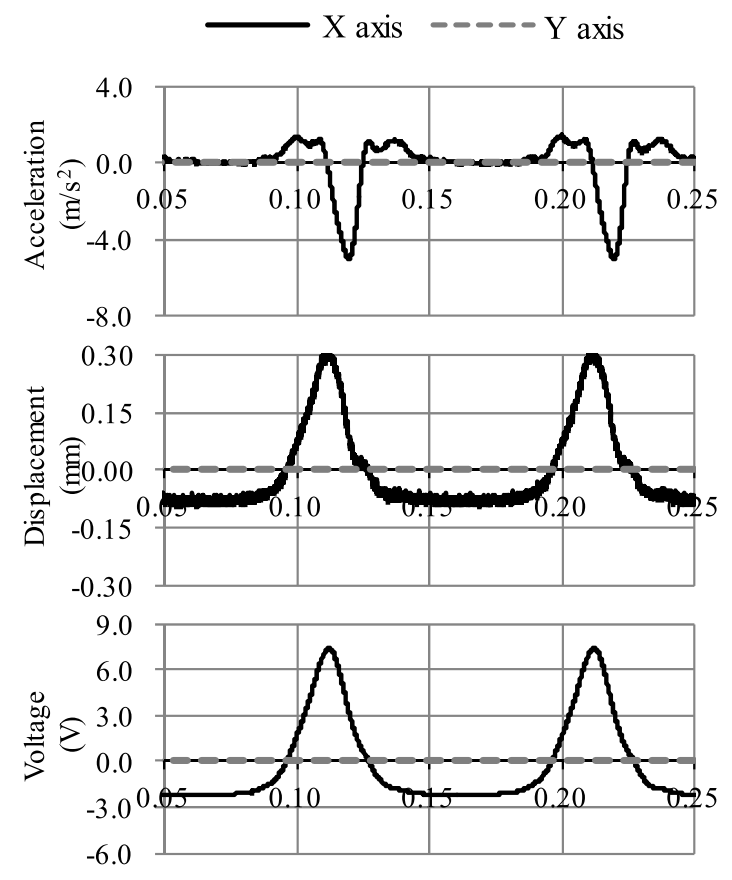

time(s)

Fig. 14. Measured results using the optimized acceleration waveform

values were calculated by the position of the mover, which were acquired with a laser displacement meter (Keyence Inc., LK-G3000, $20 \mathrm{kHz}$ sampling, $0.1 \mu \mathrm{m}$ resolution). A firstorder low-pass filter with a cutoff frequency of $50 \mathrm{~Hz}$ was applied to the acceleration waveform. Figure 14 shows the measured results of the steady-state mover acceleration, displacement and voltage when the $x$-axis was operated at a frequency of $10 \mathrm{~Hz}$ under the optimized acceleration waveform. From Fig. 14, asymmetric acceleration was also observed. The negative acceleration peaks were $5.1 \mathrm{~m} / \mathrm{s}^{2}$ and the ratio of the positive to the negative acceleration peaks were 3.7. Power consumption was $1.7 \mathrm{~W}$, which was the same as the analysis.

Finally, experiments on volunteers were conducted to confirm the effectiveness of the optimized asymmetric acceleration waveform. The experiment method and condition were the same as shown in Sect. 4.

Table 4 compares the experimental results using the
Table 4. Experimental results under various waveforms

\begin{tabular}{c|c|c|c}
\hline Waveform & $\begin{array}{c}\text { Reference } \\
(9)\end{array}$ & $\begin{array}{c}\text { Reference } \\
(11)\end{array}$ & $\begin{array}{c}\text { Optimized } \\
\text { waveform }\end{array}$ \\
\hline Ratio of peaks & 2.47 & 3.0 & 3.7 \\
\hline $\begin{array}{c}\text { Power consumption } \\
{[\mathrm{W}]}\end{array}$ & 3.2 & 2.0 & 1.7 \\
\hline $\begin{array}{c}\text { Percentage of correct } \\
\text { answers [\%] }\end{array}$ & 92 & 69 & 95 \\
\hline
\end{tabular}

optimized acceleration waveform with the results using the waveforms shown in Refs. (9) and (11). From Table 4, the optimized acceleration waveform successfully maintained a high percentage of correct answers while also further lowering the power consumption.

\section{Conclusion}

In this paper, first, the effect of the properties of an asymmetric acceleration waveform on haptic perception was investigated by conducting experiments on volunteers. Then, by applying a genetic algorithm to the optimization method, an optimized asymmetric acceleration waveform was created. Finally, the effectiveness of the optimized acceleration waveform was verified through 3-D finite element analysis and also experiments on volunteers. We were successfully able to maintain a high percentage of correct answers while further lowering the power consumption.

\section{References}

( 1 ) T. Yamaguchi, Y. Kawase, S. Suzuki, K. Hirata, T. Ota, and Y. Hasegawa: "Dynamic analysis of linear resonant actuator driven by DC motor taking into account contact resistance between brush and commutator", IEEE Trans. Magn., Vol.44, No.6, pp.1510-1513 (2008)

( 2 ) Y. Asai, K. Hirata, and T. Ota: "Dynamic analysis method of linear resonant actuator with multimovers employing 3-D finite element method", IEEE Trans. Magn., Vol.46, No.8, pp.2971-2974 (2010)

( 3 ) K. Hirata, T. Yamamoto, T. Yamaguchi, Y. Kawase, and Y. Hasegawa: "Dynamic analysis method of two-dimensional linear oscillatory actuator employing finite element method", IEEE Trans. Magn., Vol.43, No.4, pp.14411444 (2007)

( 4 ) T. Yamaguchi, Y. Kawase, K. Sato, S. Suzuki, K. Hirata, T. Ota, and Y. Hasegawa: "Trajectory analysis of 2-D magnetic resonant actuator", IEEE Trans. Magn., Vol.45, No.3, pp.1732-1735 (2009)

( 5 ) D. Ryu, G.-H. Yang, and S. Kang: "T-hive: Vibrotactile interface presenting spatial information on handle surface", in Proc. IEEE 2009 Int. Conf. Robotics and Automation, pp.683-688 (2009)

( 6 ) H. Ando, T. Miki, M. Itani, and T. Maeda: "SmartFinger: Nail-mounted tactile display", ACMSIGGRAPH 2002 Conf. Abstracts and Applications, p.78 (2002)

( 7 ) T. Amemiya and T. Maeda: "Asymmetric oscillation distorts the perceived heaviness of handheld objects", IEEE Trans. Haptics, Vol.1, No.1, pp.9-18 (2008)

( 8 ) T. Amemiya and H. Sugiyama: "Orienting kinesthetically: A haptic handheld wayfinder for people with visual impairments", ACM Trans. Accessible Comput., Vol.3, No.2, pp.1-23 (2010)

( 9 ) Y. Kono, T. Yoshimoto, and K. Hirata: "Characteristic analysis of a haptic device using a 2-DOF linear oscillatory actuator", International Journal of Applied Electromagnetics and Mechanics, 45, pp.909-916 (2014)

(10) T. Yoshimoto, K. Hirata, Y. Asai, K. Ueyama, E. Hashimoto, and Y. Takagi: "Asymmetric acceleration drive using linear oscillatory actuator under openloop control", PRZEGLAD ELEKTRO-TECHNICZNY (Electrical Review), ISSN 0033-2097, R.88 NR 7b/2012 pp.44-47 (2012)

(11) M. Kato, Y. Kono, K. Hirata, and T. Yoshimoto: "Development of a Haptic Device Using a 2-DOF Linear Oscillatory Actuator", IEEE Trans. Magn., Vol.50, No.11, 8206404 (2014) 
Masayuki Kato (Student Member) received the B.E. degree from the

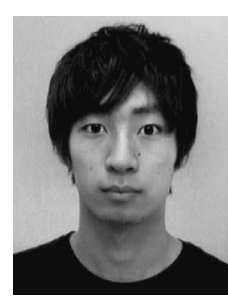
Department of Materials and Manufacturing Science, Osaka University in 2014. He is currently a student in the Department of Adaptive Machine System, Graduate School of Engineering, at Osaka University. His current research interest is on linear resonant actuators. He received Paper Award from IEEJ in 2013.

Junya Nitta (Associate) received the B.E. degree from the De-

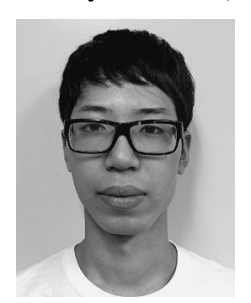
partment of Materials and Manufacturing Science, Osaka University in 2015. He is currently a student in the Department of Adaptive Machine System, Graduate School of Engineering, at Osaka University. His current research interest is on electromagnetic multidegree-of-freedom oscillatory actuators.
Katsuhiro Hirata (Senior Member) received the B.E. degree from

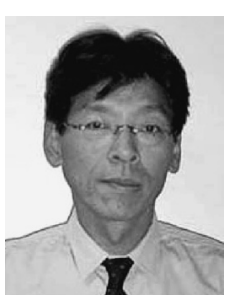
Osaka University in 1982 and D.E. degree from Doshisha University in 1996. He was a researcher at the R\&D lab., Matsushita Electric works Ltd. from 1982 to 2005. He joined Osaka University in 2005. $\mathrm{He}$ is presently a professor in the Department of Adaptive Machine Systems, Graduate School of Engineering at Osaka University. He has been engaged in research of electromagnetic applied actuators and sensors. He has received Ministry Award of Education, Science \& Technology (Ministry of Education, Culture, Sports, Science and Technology-Japan) in 2003, IEEE member. He has received OHM Technology Award (Promotion Foundation for Electrical Science and Engineering) in 2004. He received Advanced Technology Award and Paper Award (IEEJ) in 2007, 2009. 